\title{
Design and characterization of nanocarriers loaded with Levofloxacin for enhanced antimicrobial activity; physicochemical properties, in vitro release and oral acute toxicity
}

\author{
Panoraia Siafaka $^{1^{*}}$, Mehmet Evren Okur ${ }^{2}, S_{\text {Şule Ayla }}{ }^{3}$, Sevda Er $^{4}$, Emre Şefik Cağlar ${ }^{1}$, Neslihan \\ Üstündağ Okur 5
}

${ }^{1}$ Department of Pharmaceutical Technology, School of Pharmacy, Istanbul Medipol University, Istanbul, Turkey, ${ }^{2}$ University of Health Sciences, Faculty of Pharmacy, Department of Pharmacology, Istanbul, Turkey, ${ }^{3}$ Department of Histology and Embryology, School of Medicine, Istanbul Medipol University, Istanbul, Turkey, ${ }^{4}$ Yunus Emre Vocational School for Health Services, Anadolu University, Eskişehir, Turkey, ${ }^{5}$ University of Health Sciences, Faculty of Pharmacy, Department of Pharmaceutical Technology, Istanbul, Turkey

\begin{abstract}
Inorganic and carbon based nanomaterials are widely used against several diseases, such as cancer, autoimmune diseases as well as fungi and bacteria colonization. In this work, Santa Barbara Amorphous mesoporous silica (SBA), Halloysite Nanotubes (HNTs) and Multiwalled Carbon Nanotubes (CNTs) were loaded with fluoroquinolone Levofloxacin (LVF) to be applied as antimicrobial agents. The prepared via adsorption nanocarriers were characterized by Fourier-Transformed Spectroscopy, Scanning Electron Microscopy as well as High Pressure liquid Chromatography. In vitro release studies were carried out using Simulated Body Fluid at $37^{\circ} \mathrm{C}$ and data analyzed by various kinetic models showing slow dissolution over 12-24 hours. Antimicrobial studies showed improved antibacterial activity against Escherichia coli, Enterococcus faecalis, Listeria monocytogenes, Staphylococcus aureus, and Staphylococcus epidermidis compared to neat nanomaterials. CNTs were found to be the most promising candidates for LVF delivery and they were chosen to be further studied for their acute oral toxicity and histopathological examination using C57/Black mice. Histological examination depicted that drug loading did not affect mice organs morphology as well as hepatocyte degeneration, central vein degeneration and parenchymal necrosis scores. To conclude, the prepared nanomaterials present significant characteristics and can act as antimicrobial drug carriers; CNTs found to be safe candidates when orally fed to mice.
\end{abstract}

Keywords: Carbon nanotubes. Santa barbara amorphous. Halloysite nanotubes. Levofloxacin. Toxicity. Antimicrobial.

\section{INTRODUCTION}

Nanotechnology is still the emerging application field for biomedical and pharmaceutical technology applications. Nanomaterials are claimed to be promising in medical field especially as imaging agents or drug carriers due to their large surface area, improved tissue penetration, drug protection against degradation and control of drug administration (De Jong, Borm, 2008; Siafaka et al., 2016c). With the passage of time, inorganic nanoparticles consist of gold, silver or silica and carbon based nanomaterials like nanotubes, graphenes as well

\footnotetext{
*Correspondence: P.I. Siafaka. Department of Pharmaceutical Technology, School of Pharmacy, Istanbul Medipol University Istanbul, Turkey. Tel. +90216 6815100/5190, Fax: +090212 5317555. E-mail: siafpan@gmail.com iD
}

as fullerenes have been used as advanced drug delivery platforms. Graphene comprised from a mono-layer $\mathrm{sp}^{2}$-bonded carbon sheet whereas single-walled carbon nanotubes (SWCNTs) and multi-walled carbon nanotubes (MWCNTs) present a cylindrical tube shape of $\mathrm{sp}^{2}$ carbon, which roll up single- or multi-layered graphene, respectively. They display several unique physical and chemical characteristics which categorized them as suitable candidates in nanomedicine field (Siafaka et al., 2016b,c). The major concern by the CNTs use is the possible toxicity which is undesirable for biomedical applications. It has been outlined that orally fed CNTs seems to be safe even for a high dose of up to $1000 \mathrm{mg} / \mathrm{kg}$ (Kolosnjaj-Tabi et al., 2010). In most cases, CNTs have been used so as to deliver anticancer drugs (Son, Hong, Lee, 2016) or antifungal 
drugs (Pieńko et al., 2017). Although, CNTs present several properties, many researchers studied their possible use for antimicrobial applications. Generally, SWCNTs express stronger antimicrobial property than MWCNTs. The fact that synthesis of SWCNTs is more expensive than MWCNTs, imrpoving antimicrobial property of MWCNTs is mandatory. For this reason, a past study, employed the immobilization via poly(ethylene glucol) of cefalexin antimicrobial drug so as to improve the antimicrobial properties of MWCNTs (Qi et al., 2012).

Recently, nanotubes of halloysite, a low-cost clay mineral that expressed numerous physicochemical properties have been introduced as controlled release drug carrier and other biomedical applications (Hanif et al., 2016; Leporatti, 2017). In particular, halloysite nanotubes are natural green clays presenting a cylindrical structure with high aspect length-diameter ratio. Toxicity of HNTs has not been reported yet and claimed as biocompatible and thus can be used as drug delivery carriers. Antimicrobial carriers of HNTs have been reported in literature. In a recent study, HNTs were loaded with Vancomycin and further encapsulated in Alginate and Gelatin/Alginate gels as wound dressings to sustain the drug release (Kurczewska et al., 2017). Another research team evaluated the enhanced antibacterial activity against Escherichia coli incorporating halloysite nanotubes (HNTs) and silver into zinc oxide nanoparticles, since neat HNTs exhibit normal bacteria growth (Shu et al., 2017).

Silica based mesoporous materials like SBA-15 (Santa Barbara Amorphous), MCM (Mobil Composition of Matter)) and MCF (Mesoporous Cellular silica Foams) are widely found in the literature considering their large surface volume ratio as well as straight narrow channels which allow active molecules (drugs, proteins etc) to highly be absorbed into their structures (Hudson et al., 2008; Ukmar, Planinšek, 2010). In further, SBA-15 mesoporous silicas are also in a considerable interest due to their great adsorption applications such as removal and determination of many molecules. Additionally, SBA-15 cytotoxicity toward B14 fibroblasts was criticized as low (Ferenc et al., 2015). The current years, such nanostructure mesoporous materials have been investigated as drug carriers especially for anticancer drugs (Rajabi, Fayyaz, Luque, 2017), antifungal or anti-inflammatory and immunosuppressant agents (Ortiz-Bustos et al., 2017). Recently, SBA-15 loaded with Ampicillin, a known antibiotic drug was produced, expressing a burst release of the drug from the matrix (Nairi et al., 2017). Additionally, another team researched SBA-15 loaded with Vancomycin or Rifampin as well as a combination of those. They applied them against $S$. aureus and $S$. epidermidis indicating a statistically significant reduced biofilm density and fewer viable bacteria (Aguilar-Colomer et al., 2017).

In this work in order to enhance antimicrobial properties of MWCNTs, SBA-15 and HNTs, Levofloxacin (LVF) as model drug was chosen. Fluoroquinolones such as LVF have been extensively employed in genitourinary, respiratory, sinus and skin tract infections management (Siafaka et al., 2016a). Researchers have been proposed several nanocarriers for LVF loading, such as polymeric nanoparticles (Karavolos, Holban, 2016), niosomal carriers, nanofibrous matrices, thermosensitive gels (Alves et al., 2016) etc. However, to the extent of our knowledge non applications of carbon based nanomaterials, or halloysite nanotubes and SBA-15 loaded with Levofloxacin have been found.

Therefore, the aim of this work was the evaluation of these nanomaterials as oral carriers for Levofloxacin. For this purpose, SBA, HNTs and CNTs containing LVF were produced and examined for their in vitro release and antibacterial property. CNTs loaded with LVF were concluded as the best candidate was orally administered to $\mathrm{C} 57 / \mathrm{Black}$ mice to further evaluate the oral toxicity.

\section{MATERIAL AND METHODS}

\section{Material}

Pluronic P-123 triblock copolymer, tetraethyl orthosilicate (TEOS) used for SBA preparation, multiwalled Carbon Nanotubes (CNTs), Halloysite nanotubes (HNTs) and Levofloxacin (LVF) were purchased by Sigma-Aldrich Chemical Co (Steinheim, Germany). All of the used solvents and reagents were of analytical grade.

\section{Synthesis route of SBA-15}

The SBA-15 mesoporous silica was synthesized in accordance to previous reports (Karakoulia, Triantafyllidis, Lemonidou, 2008). Accordingly, Pluronic P-123 copolymer was solubilised in aqueous hydrochloric acid (HCL) $(1 \mathrm{M}, \mathrm{pH} \leq 1)$ at $35^{\circ} \mathrm{C}$. Afterwards, addition of TEOS followed and the solution was magnetically stirring for 60 minutes. The solid particles obtained by filtration, washed with distilled $\mathrm{H}_{2} \mathrm{O}$, calcinated in air $\left(550^{\circ} \mathrm{C}, 6 \mathrm{hrs}\right)$ and mesoporous silica was produced.

\section{Adsorption of the drug onto the nanocarriers}

Similarly to a previous work (Filippousi et al., 2015), $200 \mathrm{mg}$ of LVF was dissolved in $200 \mathrm{ml}$ of 
Acetonitrile/Methanol (ACN/MeOH) (10/90). Then, $200 \mathrm{mg}$ of SBA, CNTs and HNTs were added to the drug solution. The suspension was magnetic stirring under nitrogen atmosphere for $24 \mathrm{~h}$. Following the solutions were centrifuged and washed with ethanol in order to remove the unloaded LVF particles from the SBA, HNTs or CNTs surface. In order to identify the adsorption the supernatants were quantified using HPLC system and the \% adsorption was calculated, given the known LVF concentration.

\section{Characterization of the LVF loaded nanomaterials}

\section{Fourier-Transformed Infrared Spectroscopy}

The interactions between carriers and LVF drug were examined via FT-IR spectroscopy study. FTIRspectrometer (model FTIR-2000, Perkin Elmer, Turkey) was used so as to record the baseline corrected spectra, with an area ranged from 4000 to $400 \mathrm{~cm}^{-1}$, of neat nanomaterials and loaded with LVF formulations. The resolution set of $2 \mathrm{~cm}^{-1}$ ( $32 \mathrm{co}$-added scans).

\section{Scanning Electron Microscopy (SEM)}

The morphology of the pristine and LVF loaded nanomaterials was performed by using a Zeiss EVO scanning electron microscope (SEM). The accelerating voltage was $20 \mathrm{kV}$, probe current was $45 \mathrm{nA}$ and counting time was $60 \mathrm{~s}$. All the data were analyzed via Digimizer software in order to assess the mean diameter of nanocarriers.

\section{In vitro drug release}

In vitro release study of the LVF loaded nanocarriers was performed in simulated body fluid at $50 \mathrm{rpm} .100$ $\mathrm{mg}$ of the formulations were added into $100 \mathrm{~mL} \mathrm{pH} 7.4$ Simulated Body Fluid (SBF) solution (Siafaka et al., 2015 ) with a preserved temperature at $37 \pm 0.5^{\circ} \mathrm{C}$ to imitate body temperature. $0.5 \mathrm{~mL}$ of sample was removed at a predetermined time interval of 15 minute to $24 \mathrm{~h}$ while same volume of fresh medium was replaced. The analysis of the drug content was performed with HPLC. The HPLC system (HP Agilent 1100) consisted of a gradient pump and a UV detector set at $295 \mathrm{~nm}$. The used column was a $\mathrm{C} 18,120 \mathrm{~A}, 5 \mu \mathrm{m}, 250 \mathrm{~mm} \times 4.6 \mathrm{~mm}$ and column temperature was $25^{\circ} \mathrm{C}$. The mobile phase comprised from $\mathrm{H}_{2} \mathrm{O}(\mathrm{pH}=3)$ / $\mathrm{MeOH}-(60 / 6400)$. The applied injection volume was $10 \mu \mathrm{L}$. Calibration curve was constructed by using different concentrations of LVF solution (1, 2, 510, 25 and $100 \mathrm{ppm})$ obtained by dilution with mobile phase of a stock aqueous solution of 200 ppm LVF (Siafaka et al., 2016a).

\section{Analysis of release data}

In order to understand the possible release mechanism of LVF drug, the data from in vitro drug release studies was exposed to various mathematical models. The best-fit model was decided based on the highest regression values $\left(\mathrm{r}^{2}\right)$ for correlation coefficients for formulations. For the evaluation and the determination of the release mechanism, kinetic models like zero order, first order, Higuchi and Hixson Crowell were applied. The analysis was utilized to the release study of drug performed until the $60-80 \%$ of drug released. In addition, KorsemeyersPeppas equation was also used for kinetic analysis. When the mechanism is Case I (Fickian) diffusion, it is very well known that the " $n$ " value is around 0.5 (the exact value depends on the geometry). A value between 0.5 and 0.89 reveals anomalous (non-Fickian) diffusion and a value of 0.89 and above demonstrate a Case II Transport. When the release mechanism is mainly based on Fickian diffusion, a dimension less expression of the Higuchi model can be applied (Siafaka et al., 2016a).

\section{Disc diffusion method}

Escherichia coli ATCC 25922, Enterococcus faecalis ATCC 29212, Listeria monocytogenes ATCC 7644, Staphylococcus aureus ATCC 25923, and Staphylococcus epidermidis ATCC 12228 were used to evaluate antibacterial activity of formulations by performing disk diffusion method. All microorganism samples were incubated 24 hours using brain-heart infusion (BHI) broth medium at $37^{\circ} \mathrm{C}$. Following incubation, bacteria adjusted to $0.5 \mathrm{McF}$ arland turbidity standards $\left(10^{8} \mathrm{cfu} / \mathrm{mL}\right)$ with McFarland Densitometer (DEN-1, Biosan) in 0.85\% sterile saline solution. Microbial suspension was cultured on Mueller Hinton agar (MHA) medium using a swab. The entire surface of the Mueller-Hinton Agar (MHA) petri dish was covered with the required inoculum using a swab, and the petri dish was air dried for $15 \mathrm{~min}$. In a sterile environment, formulation discs were applied $5 \mu \mathrm{L}$ of every formulation were taken by forceps and placed on surface of the petri dish. 15 minutes later, dishes were incubated at $37^{\circ} \mathrm{C}$ for $24 \mathrm{~h}$. Zone diameters occurred around the discs were measured, recorded and the results were evaluated. The study was conducted as pair-parallel manner (Üstündağ-Okur et al., 2014).

\section{Acute oral toxicity study}

Animals

For this study, CNTs loaded with LVF were chosen 
as oral optimal candidate and study for their safety profile. Healthy mice were applied for the acute oral toxicity research. In fact, 21 healthy males and females C57/Black mice with weight of $25 \mathrm{~g}$ to $30 \mathrm{~g}$ and age of 8 to 10 weeks obtained from the MEDİTAM, Istanbul Medipol University. The experiments were carried out according to Declaration of Helsinki for the procedure of animals in study. The experimental procedure relating to the animals was authorized by Istanbul Medipol University Ethical committee (No.27/12/2017-77) before the study started whereas it was conducted under the internationally accepted principles for laboratory animal use and care.

\section{Acute toxicity assay}

\section{Animal conditions}

Mice located in a room at $22 \pm 2^{\circ} \mathrm{C}$ with constant humidity. A 12 light/12 dark cycle was used as commonly applied. In further, animals were acclimatized to laboratory conditions for a week prior to experiment start. They had free contact to food and water ad libitum. The mice were transferred to the laboratory $1 \mathrm{~h}$ before the test activated. All tests were completed between 09:00 and 12:00 $\mathrm{h}$ in normal room light and temperature $\left(22 \pm 1{ }^{\circ} \mathrm{C}\right)$. Mice were separated to three groups of seven animals each $(n=7)$, which were treated with the following:

Group I: Blank CNT group,

Group II: LVF loaded CNT group,

Group III: Control group (Distilled water).

Single doses of $2.8 \mathrm{mg} / \mathrm{kg}$ of blank and drug loaded CNT formulations were orally administered to both mice in the treatment groups. The formulations were suspended in a vehicle (distilled water). $0.5 \mathrm{~mL}$ of formulation was applied by oral gavage. More seven mice were administered with distilled water and named as the control groups. The mice were observed in detail for any indications of toxicity effect within the first six hours after the treatment period, and daily further for a period of 5 days. All animals were weighed and visual observations for mortality, behavioral pattern, changes in physical appearance, injury, pain and signs of illness were conducted daily during the period.

\section{Measurement of liver weights}

After 5 days period, all the mice were sacrificed and liver was isolated and examined for any lesions. In further, liver was weighed and their features were compared between both treated and control groups.

\section{Histopathological examination}

Liver obtained from the mice were transferred in 10\% neutral-buffered formaldehyde solution. After dehydration, the samples were blocked in paraffin. A microtome used to cut $5 \mu \mathrm{m}$. sections which further stained with hematoxylin and eosin. Mounted slides were evaluated under a light microscope (Nikon Eclipse Ni research microscope, Tokyo, Japan) and photographed using a digital camera (Microscope Digitale Camera DP70, Tokyo, Japan). The criteria for sections evaluation based on intensity and diffusion of degeneration and pleomorphism in hepatocytes, proliferation in bile duct, cytoplasmic eosinophily, parenchymal necrosis, degeneratin, congestion and thrombosis in central vein, and inflammation in portal space. Lastly, the counted values were summarized by each section whereas the degeneration degree was specified in accordance to a score system. Scoring was done as follow: 0-4 (no, low, moderate, high and very high, respectively as no staning $(0)$, low $(1+)$, moderate $(2+)$, heavy $(3+)$ and very heavy $(4+)$.

\section{Statistical analysis}

The statistical analysis was carried out via GraphPad Prism 7.0 software program. The results were pointed out as mean \pm standard error (SEM). Statistical significance among treated and non-treated groups was analyzed by one-way ANOVA followed by Dunnett's post hoc test. $P$ values $<0.05$ were considered statistically significant. Wherever significance has been confirmed, it is showed by $*$ p $<0.05, * *$ p $<0.01$ and $* * * p<0.001$.

\section{RESULTS AND DISCUSSION}

\section{Physicochemical characterization; FT-IR and SEM studies}

FT-IR has been evaluated as one of the most important characterization technique used in pharmaceutical technology field. This is because with FT-IR several interactions between the drug and generally active molecules could be depicted. Consequently, herein the spectra of LVF, blank and drug loaded nanocarriers examined. FT-IR spectrum of LVF (Figure 1) exhibits at $2800-3100 \mathrm{~cm}^{-1}$ characteristic peaks due to aromatic group stretching, at $1730 \mathrm{~cm}^{-1}$ the stretching of carbonyl group whereas at $1294 \mathrm{~cm}^{-1}$ stretching of amines $(\mathrm{C}-\mathrm{N})$ is also revealed. The peak at $1085 \mathrm{~cm}^{-1}$ is correlated with C-halogen group presence while at region of 3274-3415 
$\mathrm{cm}^{-1}$ hydroxyl groups of $-\mathrm{COOH}$ are illustrated (Siafaka et al., 2016a).

It has been detailed that pure SBA-15 provides silanol groups on the channel walls, which can form a weak intermolecular hydrogen bond with drugs and further allow storage of high amount of a drug and also could prolong the drug release (Jangra et al., 2016). In case of neat SBA-15, the observed broad band at $3440 \mathrm{~cm}^{-1}$ is attributed to $\mathrm{O}-\mathrm{H}$ bond stretching vibration of adsorbed water molecules, while at $1088 \mathrm{~cm}^{-1}$ can be also clearly identified the corresponding bending vibration mode (Figure 1a). For LVF loaded to SBA formulations, an alteration of the spectrum is specified. More specifically, many of the bands were shifted in lower wavenumbers due to interactions (hydrogen bonding) between the LVF and the mesoporous silica (Nanaki et al., 2017).

The peaks observed in CNTs spectra at 1063, 1625 and $3400 \mathrm{~cm}^{-1}$ are correlated with $\mathrm{C}-\mathrm{O}, \mathrm{C}=\mathrm{C}$ and $\mathrm{O}-\mathrm{H}$ bonds (due to water physically adsorbed), respectively (Figure 1a) (Farahani, Behbahani, Javadi, 2016). The band at 2980 and $2898 \mathrm{~cm}^{-1}$ is assigned to asymmetric stretching vibrations of $v\left(\mathrm{CH}_{2}\right)$. When LVF was absorbed an altered spectrum was seen due to possible interaction between LVF and the nanotubes (Filippousi et al., 2015). Moreover, the broad band at $3489 \mathrm{~cm}^{-1}$ corresponds to a higher absorbance because of LVF presence.

In case of Halloysite nanotubes the spectra is defined as similar as in the literature reported. For instance, the absorptions bands at 3697 and $3623 \mathrm{~cm}^{-1}$ are due to the stretching vibration of the inner-surface of $\mathrm{O}-\mathrm{H}$ groups of halloysite, respectively. The vibration at $1651 \mathrm{~cm}^{-1}$ depicts the interlayer water. The $1126 \mathrm{~cm}^{-1}$ peak is assigned to $\mathrm{Si}-\mathrm{O}$ stretching, while the band at $1004 \mathrm{~cm}^{-1}$ corresponds to Si-O-Si stretching vibration. The band observed at 523 $\mathrm{cm}^{-1}$ was due to the vibration of Al-O-Si. The vibration of the inner surface hydroxyl group at $908 \mathrm{~cm}^{-1}$ and $\mathrm{Si}-\mathrm{O}-\mathrm{Si}$ at $452 \mathrm{~cm}^{-1}$ confirm the existence of corresponding groups. In further, when LVF loaded to HNTs a shifting in lower wavenumbers was also observed indicating interactions between LVF and Si bands (Kurczewska et al., 2017).

SEM analysis was applied in order to morphologically examine the nanocarriers. For pristine CNTs a mean diameter of $36.8587 \pm 9.5491 \mathrm{~nm}$ was depicted. When LVF was absorbed onto these nanocarriers no morphological alterations was observed whereas drug crystals have not been identified. Given that, LVF drug was not crystallized and thus an increased release rate is found.

SBA-15 mesoporous silicas particles are comprised from tubular mesopores in an ordered hexagonal array (Karakoulia, Triantafyllidis, Lemonidou, 2008). SEM study of the prepared SBA-15 indicated the appearance of a cluster of particles with mean diameter $449.5 \pm 107.4 \mathrm{~nm}$, which are aggregated forming larger clusters of 4-5 $\mu \mathrm{m}$.
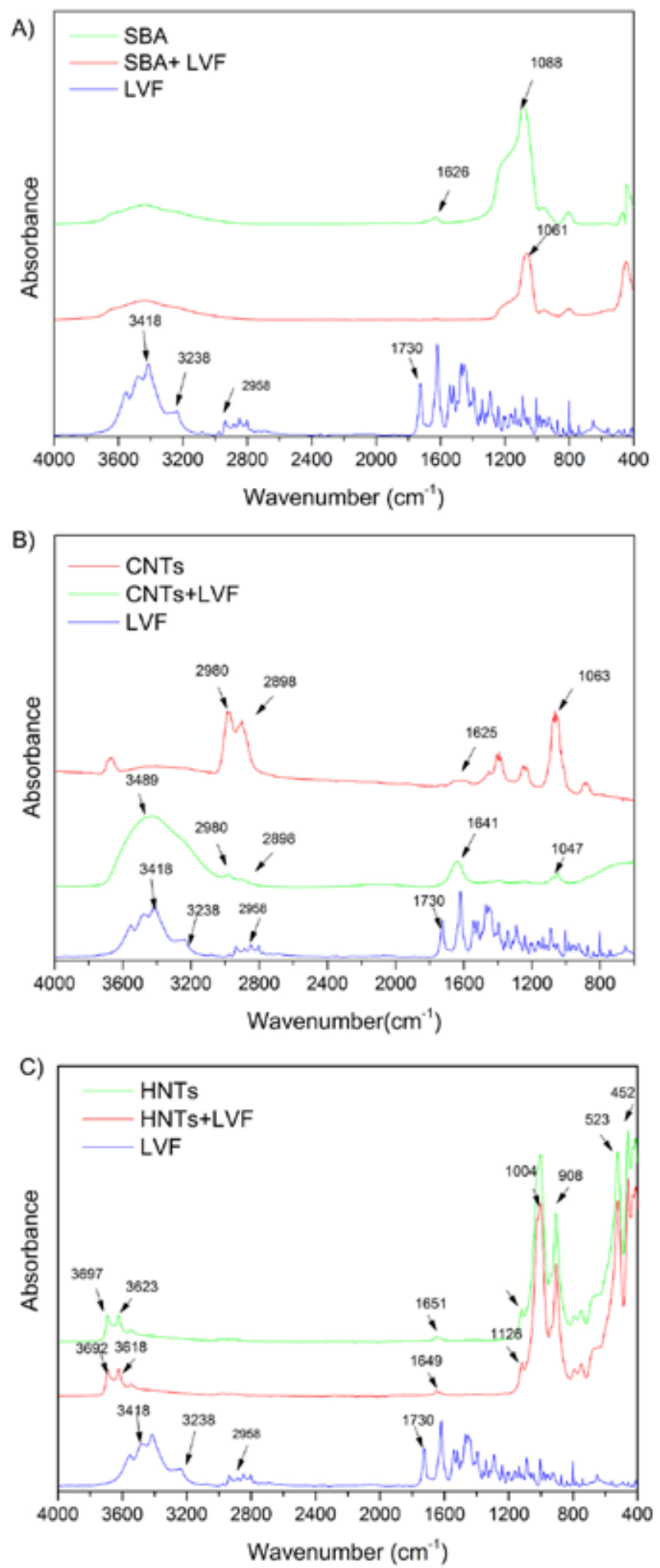

FIGURE 1 - FT-IR spectroscopy studies for A) SBA-15 neat and loaded with LVF B) CNTs neat and loaded with LVF and C) HNTs neat and loaded with LVF. 
When LVF loaded to the SBA-15 a more compact form was achieved probably due to the drug loading. However, drug molecules were not observed indicating that drug did not crystallize. In case of HNTs a cluster of tubular shaped particles is investigated. As follows, tubes with a mean diameter of $99.18 \pm 26.31 \mathrm{~nm}$ are observed. Nonetheless, the cluster of these particles presents a diameter of $2 \mu \mathrm{m}$. To conclude, when the drug was absorbed the HNTs structure did not affected.

\section{In vitro release and data analysis}

It has been reported that Levofloxacin is hydrophilic drug. In most cases, hydrophilic drugs express high drug loading however in this work, the drug loading is mostly accelerating by other parameters. When nanomaterials with high surface area ratios as CNTs, HNTs or SBA are used also drug loading is believed to be high. Accordingly, herein LVF was dissolved using ACN/MeOH solution and nanomaterials were dispersed in the solution in order to obtain high drug loading capacity. The isolation of the materials was achieved via centrifugation and the supernatants were quantified using HPLC in order to find LVF concentration. Table I exhibited the results of the LVF adsorbed nanomaterials. It can be concluded that SBA presents the higher drug loading (23.64\%) possibly because of the interactions between LVF and silanol groups, following by CNTs $(10.1 \%)$ whereas HNTs absorbed only $2.6 \%$ of the drug.

Currently, Levofloxacin has been approved as oral, ocular or injectable systems by FDA. LVF is a highly hydrophilic active pharmaceutical ingredient (API) and for this reason was fully dissolved within the first $2 \mathrm{~h}$ (data are not shown). However, when the drug is absorbed in the nanocarriers, a slower release rate is observed (Figure 3 ). It is reported that incorporating a hydrophilic drug onto

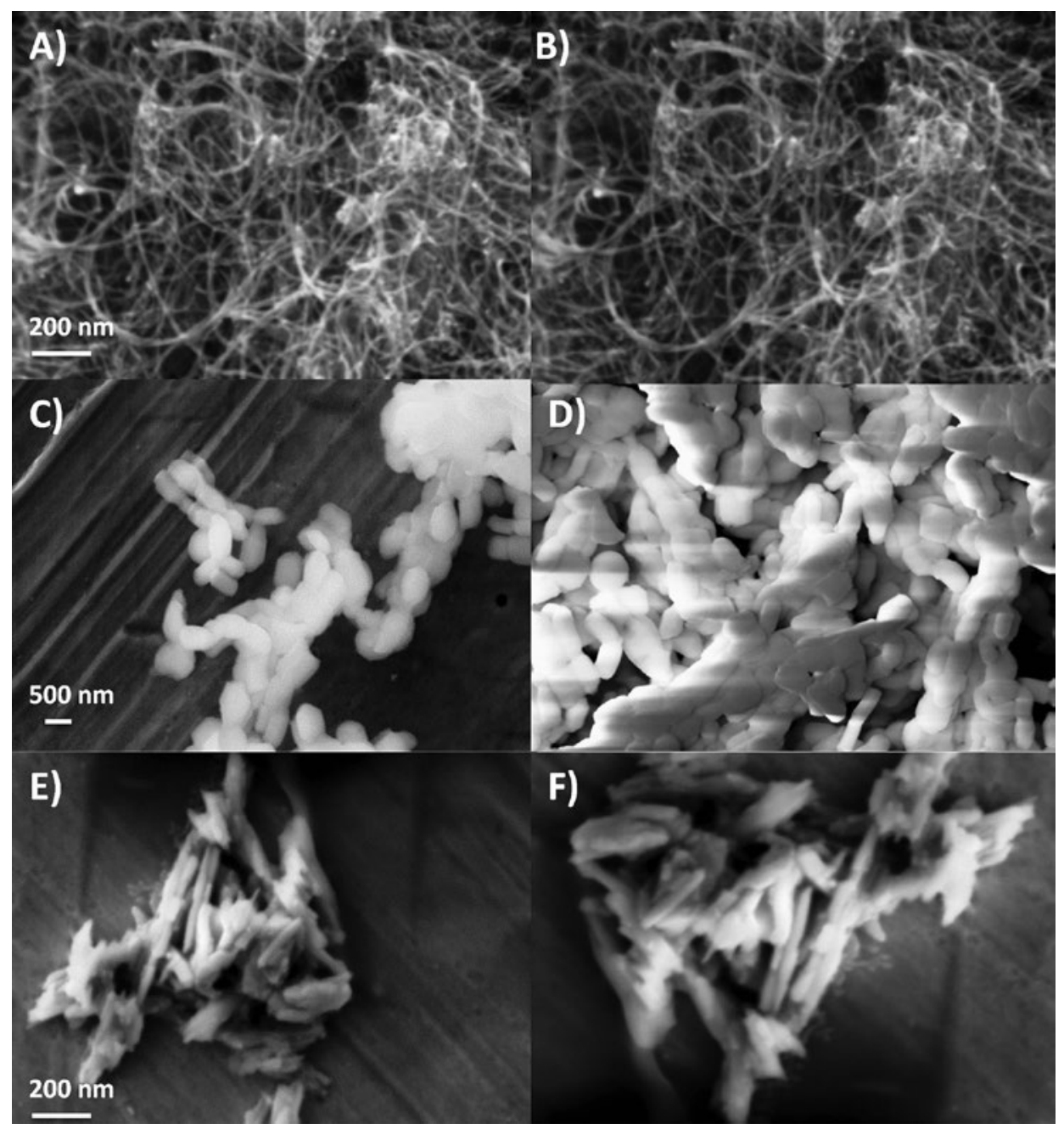

FIGURE 2 - SEM analysis for A) pure CNTs (original magnification valueX100), B) CNTs loaded with LVF(original magnification valueX100), C) pure SBA-15(original magnification valueX50), D) SBA-15 loaded with LVF(original magnification valueX50), E) pure HNTs (original magnification valueX55), and F) HNTs loaded with LVF(original magnification valueX55). 
TABLE I - Levofloxacin concentration (\%) at filtrates and adsorbed into nanomaterials quantified by HPLC

\begin{tabular}{lccc}
\hline & CNTs & SBA & HNTs \\
\hline Levofloxacin theoretical organic solution concentration $(\mathrm{mg} / \mathrm{mL})$ & 1 & 1 & 1 \\
\hline Levofloxacin quantified at filtrate $(\%)$ & 89.9 & 76.36 & 97.4 \\
\hline Levofloxacin adsorbed in nanomaterials $(\%)$ & 10.1 & 23.64 & 2.6 \\
\hline
\end{tabular}

nanomaterials, porous or not, a slower release rate can be achieved. Nonetheless, in almost all cases a burst effect is accomplished (Filippousi et al., 2016; Filippousi et al., 2015).

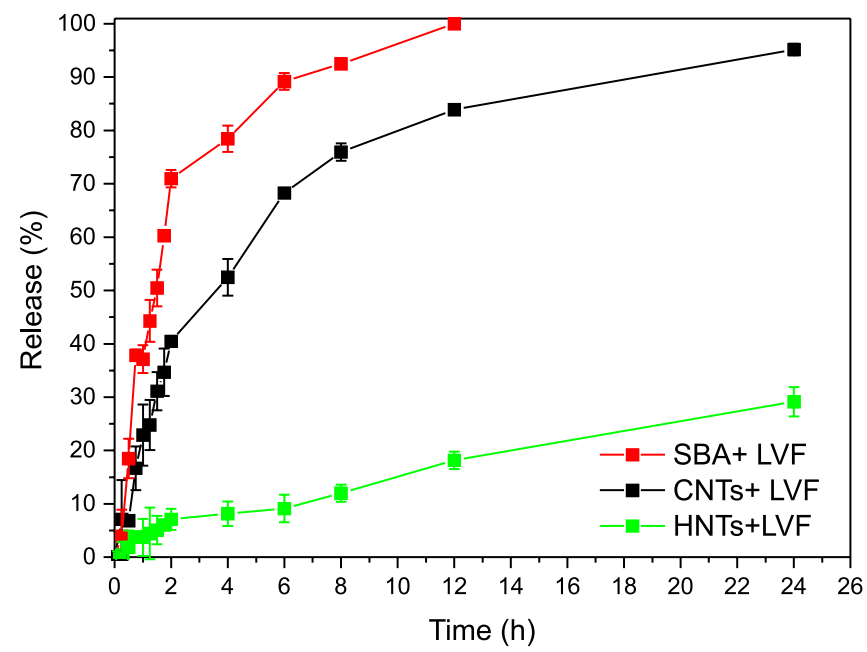

FIGURE 3 - In vitro release studies of LVF from SBA-15, CNTs and HNTs.

In this study, a low burst phenomenon can be seen for all the nanocarriers, which can be interpreted in regards to the drug deposition on the surface of the nanocarriers. After the initial immediate release, a slower rate is observed. For instance, in case of SBA a more vigorous rate can be observed and $100 \%$ of the drug is released in 12 hours. In detail, in 1 hour $37 \%$ of LVF was dissolved whereas after 2 hours of dissolution, SBA released almost the $70 \%$ of LVF. Finally, 12 hours later complete dissolution is observed. Among others, SBA-15 can control the drug release due to its gate like scaffold nature in pure and in functionalized form (Jangra et al., 2016). This reduce on dissolution rate is important since an immediate high drug dosage can be toxic to the living organism and thus controlling the rate and administration can be very effective.

Likewise, in case of carbon nanotubes more sustained release is depicted. For instance, after 1 hour almost $23 \%$ of LVF was dissolved, in 2 hours dissolution reached at $40 \%$ and complete release was achieved after 24 hours. Compared to these nanocarriers, HNTs dissolution profile is completely different. In fact, the first 2 hours of examination only $7 \%$ of the drug was released and in 24 hours release rate reached $30 \%$. These observations are rational given that HNTs are used in the literature as controlled release formulations (Cavallaro et al., 2017).

Many past studies examine release mechanism of nanomaterials by various kinetic models. In most of the studies polymeric nanoparticles are evaluated for their mechanism. Diffusion and erosion are the main release mechanisms, found in drug delivery systems. In this work, the data from in vitro release studies of formulations were evaluated according to the zero order, first order, Higuchi and Hixson Crowell and Korsmeyer-Peppas kinetic models (Table II). According to the analysis of all mathematical models, it is clearly seen that first order model showed obviously a better fit for all formulations. It has been reported that first-order model is fitted in porous materials loaded with aqueous soluble drugs (Ortiz-Bustos et al., 2017).

Furthermore, to evaluate in vitro release kinetics of LVF, the Korsmeyer-Peppas model also examined for LVF loaded formulations. When $\mathrm{n}=1$, the release rate is non-dependent of time (zero-order) (case II transport). In

TABLE II - Kinetic data of LVF release parameters obtained from formulations.

\begin{tabular}{lccccccccccccccc}
\hline Models & \multicolumn{2}{c}{ Zero order } & \multicolumn{3}{c}{ First order } & \multicolumn{3}{c}{ Higuchi } & \multicolumn{3}{c}{ Hixson-Crowell } & \multicolumn{2}{c}{$\begin{array}{c}\text { Korsmeyer- } \\
\text { Peppas }\end{array}$} \\
\hline Samples & $\mathbf{r}^{\mathbf{2}}$ & $\mathbf{n}$ & $\mathbf{m}$ & $\mathbf{r}^{\mathbf{2}}$ & $\mathbf{n}$ & $\mathbf{m}$ & $\mathbf{r}^{\mathbf{2}}$ & $\mathbf{n}$ & $\mathbf{m}$ & $\mathbf{r}^{\mathbf{2}}$ & $\mathbf{n}$ & $\mathbf{m}$ & $\mathbf{r}^{\mathbf{2}}$ & $\mathbf{n}$ \\
\hline CNTs+LVF & 0.74 & 24.70 & 3.796 & 0.975 & 4.422 & -0.13 & 0.922 & 2.34 & 22.266 & 0.913 & 0.364 & 0.124 & 0.924 & 0.79 \\
SBA+LVF & 0.74 & 29.05 & 9.775 & 0.954 & 4.395 & -0.32 & 0.880 & 2.01 & 36.380 & 0.899 & 0.421 & 0.320 & 0.915 & 1.27 \\
HNTs+LVF & 0.97 & 2.863 & 1.138 & 0.982 & 4.579 & -0.01 & 0.965 & -2.59 & 5.991 & 0.979 & 0.042 & 0.020 & 0.883 & 0.76 \\
\hline
\end{tabular}


case of $\mathrm{n}=0.5$ a fickian diffusion is declared but when $0.5<\mathrm{n}<1.0$ both means diffusion and non-fickian transport are involved. Lastly, in case of $n>1.0$ super case II transport is apparent ' $\mathrm{n}$ ' is the slope value of $\log \mathrm{mt} / \mathrm{m} \infty$ versus log time curve (Üstündağ-Okur et al., 2015).

In this study, CNTs and HNTs formulations exhibited anomalous (non-Fickian transport) diffusion mechanism with "n" values 0.794 and 0.763 , respectively (Table II). The " $n$ " value of SBA formulation has been found to be more than 1 . This result indicates that the drug release from the matrix suggests super Case II transport given that these values are characteristic for mass transfer following a non-Fickian model, suggesting that the diffusion of the drug is restricted by the interactions among the drug and the surface silica species (Ortiz-Bustos et al., 2017).

\section{Antibacterial activity of LVF formulations}

Levofloxacin, as it has been reported is a broad spectrum antibacterial agent used to treat numerous bacterial infections such as acute bacterial sinusitis, pneumonia, urinary tract infections, chronic prostatitis, and some types of gastroenteritis (Siafaka et al., 2016a). In addition, it can be used along with other antibiotics to manage tuberculosis, meningitis, or pelvic inflammatory disease. It has been reported that this fluoroquinolone derivative presents high bactericidal activity against $M$. Tuberculosis, P. aeruginosa, S. Aureus, Escherichia coli etc.

Herein, the antimicrobial activity of LVF loaded nanocarriers (Table III) was tested against four opportunistic pathogens commonly associated with oral and pulmonary disease: Staphylococcus aureus, Staphylococcus epidermidis, Enterococcus faecalis, Listeria monocytogenes. As it was expected the pristine nanocarriers did not express any bactericidal activity, although it has been claimed that carbon nanotubes could express antimicrobial activity. However, an inhibition growth was seen from HNTs to E.coli strain $(8 \mathrm{~mm})$. In the literature, neat HNTs did not exhibit any bactericidal activity used in its pristine form but when it was decorated with Ag or $\mathrm{ZnO}$ showed improved properties (Shu et al., 2017). Statistically significant difference has not been pointed out for antibacterial activity between CNT and SBA against Staphylococus epidermidis, Escherichia coli, Staphylococus aureus, Listeria monocytogenes ( $\mathrm{p}$ $>0.05$ ). Zone inhibition diameters of LVF solution were determined as $22 \mathrm{~mm}$ against Staphylococus epidermidis and Listeria monocytogenes (Figure 4).

Moreover, a similar inhibition halo (20-32 mm) was observed in case of SBA-15 and MWCNTs loaded with Levofloxacin for all the studied strains revealing that antibiotic activity of such carriers is promising. For example, when SBA loaded LVF was applied against E. coli a higher inhibition halo $(32 \mathrm{~mm})$ was found compared to CNTs loaded LVF $(28 \mathrm{~mm})$ which however is not statistically significant. Nonetheless, CNTs loaded with LVF was superior against $\mathrm{S}$. epidermidis, $\mathrm{S}$. aureus and E. faecalis presenting 22, 28, 22 and $32 \mathrm{~mm}$ inhibition zone, respectively. SBA loaded LVF and CNTs loaded LVF showed same bactericidal ability in case of Listeria monocytogenes $(20 \mathrm{~mm})$.

It has been revealed, that CNTs immobilized with Cefalexin exhibited also improved bactericidal activity (Qi et al., 2012). Similarly, a past study evaluated SBA15 loaded with Ag demonstrating increased antimicrobial activity against $\mathrm{E}$. coli due to a synergistic action between extracellular ROS and the toxicity of $\mathrm{Ag}^{+}$, which induced intracellular ROS production and subsequent cell death (Wang et al., 2014). In case of HNTs, a slight decreased inhibition halo is seen which is associated with the controlled drug release of the nanocarriers. It can be concluded that compared to free LVF solution a $5 \%$

TABLE III - Results of disc diffusion tests (mm)

\begin{tabular}{ccccccc}
\hline & \multicolumn{5}{c}{ Microorganisms } \\
\hline \multirow{2}{*}{ Formulations } & $\begin{array}{c}\text { Staphylococcus } \\
\text { epidermidis }\end{array}$ & $\begin{array}{c}\text { Escherichia } \\
\text { coli }\end{array}$ & $\begin{array}{c}\text { Staphylococcus } \\
\text { aureus }\end{array}$ & $\begin{array}{c}\text { Listeria } \\
\text { monocytogenes }\end{array}$ & $\begin{array}{c}\text { Enterococcus } \\
\text { faecalis }\end{array}$ \\
\cline { 2 - 7 } & $\mathrm{SBA}$ & 0 & 0 & 0 & 0 & 0 \\
& $\mathrm{SBA}+\mathrm{LVF}$ & 20 & 32 & 18 & 20 & 24 \\
$\mathrm{C}$ & $\mathrm{CNTs}$ & 0 & 0 & 0 & 0 & 0 \\
\hline
\end{tabular}


MICROORGANISMS

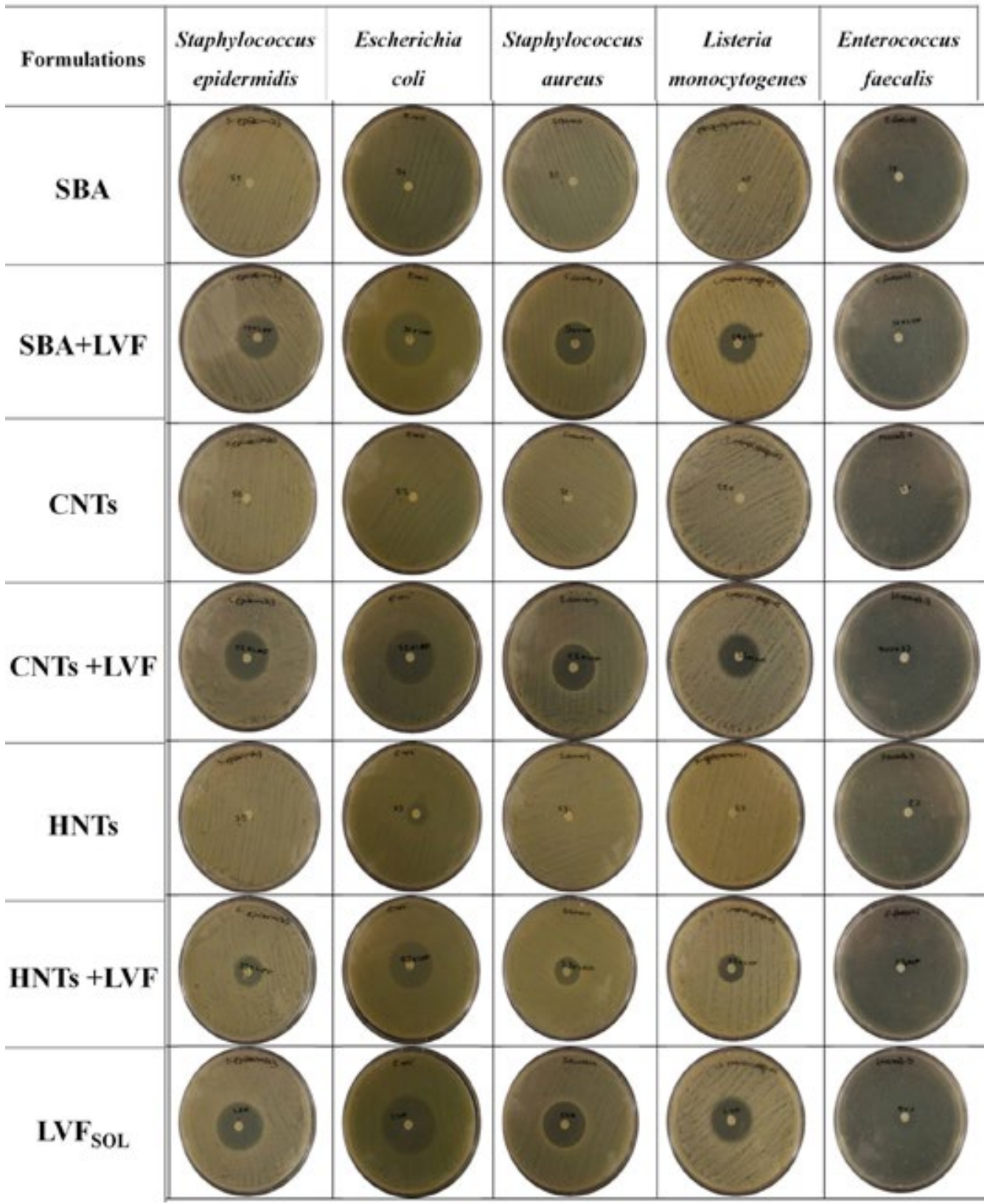

FIGURE 4 - Antibacterial activity of the blank and LVF loaded formulations.

reduction in the inhibition zone is seen, which it was expected due to the slower release rate and the difficulty of the drug to be diffused from the carrier (Islan et al., 2016). For the oral toxicity experiment test, CNTs formulations were chosen as the most promising formulations since they also showed better antibacterial activity properties.

\section{Oral acute toxicity}

From a scientific aspect, toxicity is the degree to which chemicals or other toxic substances can affect a living organism. Toxicity can have an impact either on a whole organism (animal, bacteria, and humans) but can also affect a substructure of the organism, such as cells (cytotoxicity) or a bodily organ like the liver (hepatotoxicity) or kidney (nephrotoxicity). Fortunately, living organisms can defend themselves against chemical exposure; for instance, via metabolism, distribution, and excretion various adverse effects can be determined and the risk can be overcome. Nonetheless, exposure in chemicals and drugs, due to renal clearance and detoxification mainly lead to kidney and liver injury (Björnsson, 2016). 
As it was referred in this work, CNTs blank and loaded with Levofloxacin were studied for their acute oral toxicity. Throughout literature, CNTs toxicological study reveals conflicting results. Some preliminary in vitro toxicological tests suggested that CNTs are toxicologically benign to certain cells, whereas other studies showed that CNTs, mostly raw materials have the potential of being dangerous to many organisms (He et al., 2013). In particular, although CNTs cytotoxicity is not always revealed by the cell culture assay, oxidative stress is the main cause of cytotoxicity (Patlolla, McGinnis, Tchounwou, 2011).

The available safety data indicate that CNTs are of low toxicity via various exposure pathways for biomedical applications. In further, Pulskamp, Diabate and Krug (2007) demonstrated that CNTs did not express acute toxicity in pulmonary epithelial and macrophage cell lines (Pulskamp, Diabate, Krug, 2007). Additionally, an important parameter is the administration dose of CNTs. The most difficult part on CNT toxicokinetics examination is to compare the currently published data due to the differences among the materials. Parameters such as size, dispersibility, functionalization, and metal catalyst contaminants, can lead to discrepancies among toxicity results. Subsequently, detailed chemical and structural characterization of the nanomaterial has to always be given in toxicokinetics assessments of CNT.

In this study, the mice from control and treated groups were orally fed with vehicles, blank and LVF loaded CNTs, respectively. A daily monitoring of the mice was done until $5^{\text {th }}$ day in order to observe any toxic signs and mortality. It can be safely reported that toxic symptoms or mortality were not revealed for all animal groups which lived up to 5 days of the experimental period after treated with blank and drug loaded formulations using a single dose level of 2.8 $\mathrm{mg} / \mathrm{kg}$ body weight. During toxicity evaluation the observation of any clinical symptoms is one of the most significant parameter (Jothy et al., 2011). Mice behavioral patterns were evaluated the first $6 \mathrm{~h}$ and 12 $\mathrm{h}$ after the administration. It was found that animals in control and CNTs treated groups were normal whereas their behavior, skin, eyes, mucous membrane, sleep, diarrhea, breathing, impairment in food intake and water consumption, postural abnormalities and hair loss have not shown any inconsistencies. Even more, body and liver weight was similar. In detail, in this study liver was not adversely affected throughout the treatment. The liver weight of mice between blank and drug loaded CNTs treated and control groups did not show any statistically significant differences $(\mathrm{P}>0.05)$ (Figure 5).

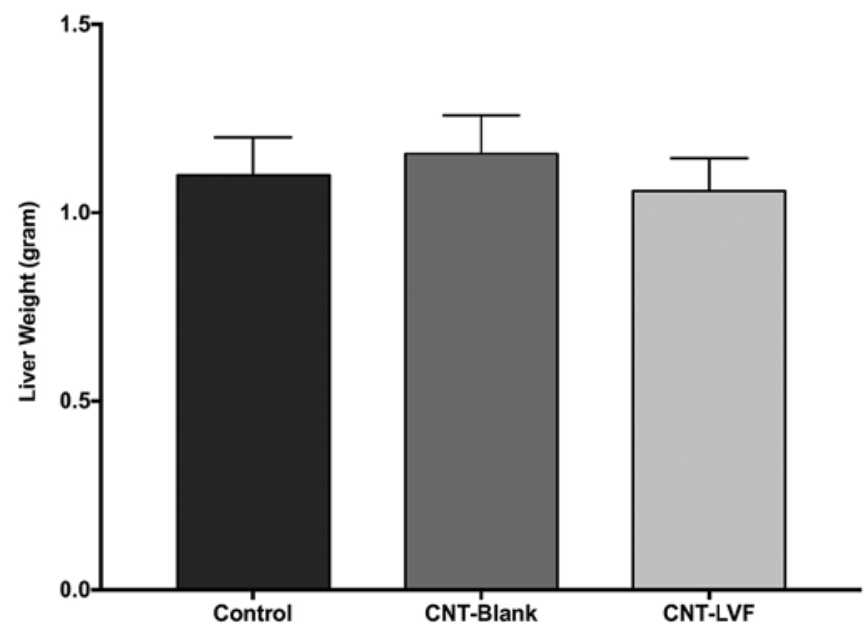

FIGURE 5 - Comparison of liver weight of mice among groups. Statistically significant as compared to control; $\mathrm{P}<0.05(*)$, $\mathrm{P}<0.01(* *), \mathrm{P}<0.001(* * *)$.

Furthermore, histological examination was performed so as to investigate any alteration in cell structure of the organs. It is well reported that histological study is significant for the evaluation of pathological changes in tissues and organs OECD/OCDE, 2008). In the present study, histopathological assessment of acute oral ingestion of formulations specified that the formulations did not adversely show impact in the morphology of mice organs. Further, macroscopic investigation of mice organs treated with CNTs did not show any alterations in color compared to control. Finally, autopsy at the end of the experiment did not point out obvious alterations in the liver from both control and treated mice. Figure 5 illustrates the microscopic structures of the organs implying unnoticeable differences between the control and treated mice groups. Likewise, CNTs treated mice organ did not display any changes in cell structure or any unfavorable effects when studied under the light microscope using multiple magnification power. It can be claimed that the structure or coordination of cells in CNTs treated organs are more or less similar compared with the control group organs.

Table IV shows hepatocyte degeneration, cytoplasmic eosinophilia, central vein degeneration and parenchymal necrosis scores of mice livers after treatment 5 days with formulations and control group. All hepatocyte degeneration, cytoplasmic eosinophilia, central vein degeneration and parenchymal necrosis scores were found less than 1. Hepatocyte degeneration and parenchymal necrosis of blank and drug loaded CNTs were found as 0.571. Blank and LVF loaded CNTs scores present same scores except cytoplasmic eosinophilia scores. It can be pointed out that the drug loading have not shown any effect 
TABLE IV - Hepatocyte degeneration, cytoplasmic eosinophilia, central vein degeneration and parenchymal necrosis scores of the formulations after treatment 5 days

\begin{tabular}{lccc}
\hline Groups & Control & CNT-Blank & LVF loaded CNT \\
\hline Hepatocyte degeneration & 0 & $0.571 \pm 0.202$ & $0.571 \pm 0.202$ \\
Cytoplasmic eosinophilia & 0 & $0.714 \pm 0.184$ & $0.857 \pm 0.261$ \\
Central vein degeneration & 0 & $0.429 \pm 0.202$ & $0.429 \pm 0.202$ \\
Parenchymal necrosis & 0 & $0.571 \pm 0.202$ & $0.571 \pm 0.202$ \\
\hline
\end{tabular}

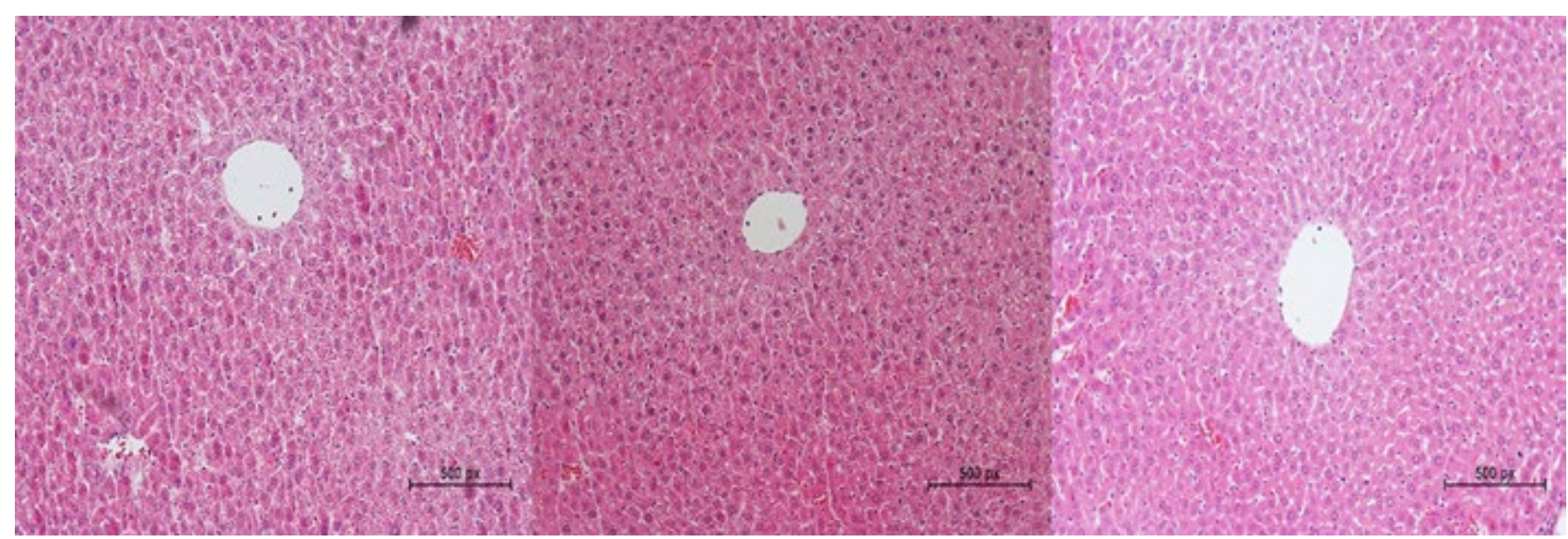

FIGURE 6 - Histological examination of liver A) blank CNTs B) LVF loaded CNTs C) Distilled water (control group) $20 \mathrm{x}$ (magnification value).

on hepatocyte degeneration, central vein degeneration and parenchymal necrosis scores.

\section{CONCLUSION}

This study aims to check suitability of CNTs, HNTs and SBA carriers as oral drug delivery pltaforms. The prepared LVF loaded formulations showed improved antibacterial property against several strains. FTIR spectroscopy revealed interactions between the nanocarriers and the LVF drug while SEM images indicate slight alteration of their morphology after drug loading in case of SBA carriers. From the preliminary studies, LVF loaded CNTs were selected as best formulation. Histological examination of CNTs loaded with LVF showed that drug loading did not affect the hepatocyte degeneration, central vein degeneration and parenchymal necrosis scores. In further, histopathological examination of acute oral ingestion of formulations illustrated that the formulations did not reveal adversely effects on the morphology of mice organs. To conclude, the above nanomaterials showed interesting characteristics and could be an alternative option as antimicrobial drug carriers in biomedical applications.

\section{CONFLICT OF INTEREST}

No declaration.

\section{ACKNOWLEDGEMENTS}

The authors would like to acknowledge Istanbul Medipol University MEDITAM for enabling us to use its laboratory instruments.

\section{REFERENCES}

Aguilar-Colomer, A Doadrio JC, Pérez-Jorge C, Manzano M, Vallet-Regí M, Esteban J, et al. Antibacterial effect of antibioticloaded SBA-15 on biofilm formation by Staphylococcus aureus and Staphylococcus epidermidis. J Antibiot (Tokyo). 2017;70(3):259-263.

Alves DA, Machado D, Melo A, Pereira RFC, Severino P, Hollanda LM, Araújo DR, Lancellotti M. Preparation of thermosensitive gel for controlled release of levofloxacin and their application in the treatment of multidrug-resistant bacteria. Biomed Res Int. 2016;2016:9702129. 
Björnsson E. Hepatotoxicity by drugs: the most common implicated agents. Int J Mol Sci. 2016;17(2):224.

Cavallaro G, Danilushkina A, Evtugyn V, Lazzara G, Milioto S, Parisi F, et al. Halloysite nanotubes: controlled access and release by smart gates. Nanomaterials. 2017;7(8):E199.

De Jong WH, Borm PJA. Drug delivery and nanoparticles:applications and hazards. Int J Nanomedicine. 2008;3(2):133-149.

Farahani BV, Behbahani GR, Javadi N. Functionalized multi walled carbon nanotubes as a carrier for doxorubicin: drug adsorption study and statistical optimization of drug loading by factorial design methodology. J Braz Chem Soc. 2016;27(4):694-705.

Ferenc M, Katir N, Milowska K, Bousmina M, Majoral J-P, Bryszewska M, El Kadib A. Haemolytic activity and cellular toxicity of SBA-15-type silicas: Elucidating the role of the mesostructure, surface functionality and linker length. J Mater Chem B. 2015;13:2714-2724.

Filippousi M, Siafaka PI, Amanatiadou EP, Nanaki SG, Neratzaki M, Bikiaris DN, Vizirianakis IS, Van Tendeloo G. Modified chitosan coated mesoporous strontium hydroxyapatite nanorods as drug carriers. J Mater Chem B. 2015;3(29):59916000.

Filippousi M, Turner S, Leus K, Siafaka PI, Tseligka ED, Vandichel M, et al. Biocompatible Zr-based nanoscale MOFs coated with modified poly( $\varepsilon$-caprolactone) as anticancer drug carriers. Int J Pharm. 2016;509(1-2):208-218.

Hanif M, Jabbar F, Sharif S, Abbas G, Farooq A, Aziz M. Halloysite nanotubes as a new drug-delivery system: a review. Clay Miner. 2016;51(3):469-477.

He H, Pham-Huy LA, Dramou P, Xiao D, Zuo P, Pham-Huy C. Carbon nanotubes: Applications in pharmacy and medicine. Biomed Res Int. 2013;2013:578290.

Hudson SP, Padera RF, Langer R, Kohane DS. The biocompatibility of mesoporous silicates. Biomaterials. 2008;29(30):4045-55.

Islan GA, Tornello PC, Abraham GA, Duran N, Castro GR. Smart lipid nanoparticles containing levofloxacin and DNase for lung delivery. Design and characterization. Colloids Surfaces B Biointerfaces. 2016;143:168-176.
Jangra S, Jangra S, Devi S, Tomer VK, Chhokar V, Duhan S. Improved antimicrobial property and controlled drug release kinetics of silver sulfadiazine loaded ordered mesoporous silica. J Asian Ceram Soc. 2016;4(3):282-288.

Jothy SL, Zakaria, Z, Chen Y, Lau YL, Latha LY, Sasidharan S. Acute oral toxicity of methanolic seed extract of cassia fistula in mice. Molecules. 2011;16(6):5268-5282.

Karakoulia SA, Triantafyllidis KS, Lemonidou AA. Preparation and characterization of vanadia catalysts supported on nonporous, microporous and mesoporous silicates for oxidative dehydrogenation of propane (ODP). Microporous Mesoporous Mater. 2008;110(1):157-166.

Karavolos M, Holban A. Nanosized drug delivery systems in gastrointestinal targeting: Interactions with microbiota. Pharmaceuticals. 2016;9(4):1-15.

Kolosnjaj-Tabi J, Hartman KB, Boudjemaa S, Ananta JS, Morgant G, Szwarc H, et al. In vivo behavior of large doses of ultrashort and full-length single-walled carbon nanotubes after oral and intraperitoneal administration to swiss mice. ACS Nano. 2010;4(3):1481-1492.

Kurczewska J, Pecyna P, Ratajczak M, Gajęcka M, Schroeder G. Halloysite nanotubes as carriers of vancomycin in alginatebased wound dressing. Saudi Pharm J. 2017;25(6):911-920.

Leporatti S. Halloysite clay nanotubes as nano-bazookas for drug delivery. Polym Int. 2017; 66(8):1111-1118.

Nairi V, Medda L, Monduzzi M, Salis A. Adsorption and release of ampicillin antibiotic from ordered mesoporous silica. J Colloid Interface Sci. 2017;497:217-225.

Nanaki S, Siafaka PI, Zachariadou D, Nerantzaki M, Giliopoulos DJ, Triantafyllidis KS, et al. PLGA/SBA-15 mesoporous silica composite microparticles loaded with paclitaxel for local chemotherapy. Eur J Pharm Sci. 2017;99:32-44.

OECD/OCDE. Test No. 407: Repeated Dose 28-day Oral Toxicity Study in Rodents. OECD Publishing; 2008.

Ortiz-Bustos J, Martín A, Morales V, Sanz R, García-Muñoz RA. Surface-functionalization of mesoporous SBA-15 silica materials for controlled release of methylprednisolone sodium hemisuccinate: Influence of functionality type and strategies of incorporation. Microporous Mesoporous Mater. 2017;240:236245. 
Patlolla A, McGinnis B, Tchounwou P. Biochemical and histopathological evaluation of functionalized single-walled carbon nanotubes in Swiss-Webster mice. J Appl Toxicol. 2011;31(1):75-83.

Pieńko T, Grudzień M, Taciak PP, Mazurek AP. Adsorption of antifungal drugs inside pristine and functionalized fullerenes and nanotubes: DFT investigation. Curr Comput Aided Drug Des. 2017;13(3):177-185.

Pulskamp K, Diabate S, Krug H. Carbon nanotubes show no sign of acute toxicity but induce intracellular reactive oxygen species in dependence on contaminants. Toxicol Lett. 2007;168(1):5874.

Qi X, Gunawan P, Xu R, Chang MW. Cefalexin-immobilized multi-walled carbon nanotubes show strong antimicrobial and anti-adhesion properties. Chem Eng Sci. 2012;84:552-556.

Rajabi F, Fayyaz F, Luque R. Cytosine-functionalized SBA15 mesoporous nanomaterials: Synthesis, characterization and catalytic applications. Microporous Mesoporous Mater. 2017;253:64-70.

Shu Z, Zhang Y, Yang Q, Yang H. Halloysite nanotubes supported $\mathrm{Ag}$ and $\mathrm{ZnO}$ nanoparticles with synergistically enhanced antibacterial activity. Nanoscale Res Lett. 2017;12(1):135.

Siafaka P, Betsiou M, Tsolou A, Angelou E, Agianian B, Koffa $\mathrm{M}$, et al. Synthesis of folate- pegylated polyester nanoparticles encapsulating ixabepilone for targeting folate receptor overexpressing breast cancer cells. J Mater Sci Mater Med. 2015;26(12):275.

Siafaka PI, Zisi AP, Exindari MK, Karantas ID, Bikiaris DN. Porous dressings of modified chitosan with poly(2-hydroxyethyl acrylate) for topical wound delivery of levofloxacin. Carbohydr Polym. 2016a;143:90-99.
Siafaka PI, Barmbalexis P, Bikiaris DN. Novel electrospun nanofibrous matrices prepared from poly(lactic acid)/ poly(butylene adipate) blends for controlled release formulations of an anti-rheumatoid agent. Eur J Pharm Sci. 2016b;88:12-25.

Siafaka PI, Üstündağ Okur N, Karavas E, Bikiaris, DN. Surface modified multifunctional and stimuli responsive nanoparticles for drug targeting: Current status and uses. Int J Mol Sci. 2016c;17(9):pii E1440.

Son KH, Hong JH, Lee JW. Carbon nanotubes as cancer therapeutic carriers and mediators. Int J Nanomed. 2016;11:51635185.

Ukmar T, Planinšek O. Ordered mesoporous silicates as matrices for controlled release of drugs. Acta Pharm. 2010;60(4):373385.

Üstündağ-Okur N, Ege MA, Karasulu HY, et al. Preparation and characterization of naproxen loaded microemulsion formulations for dermal application. Int J Pharm. 2014;4(4):3342.

Üstündağ-Okur N, Gökçe EH, Bozbıyık Dİ, Eğrilmez S, Ertan G, Özer Ö. Novel nanostructured lipid carrier-based inserts for controlled ocular drug delivery: evaluation of corneal bioavailability and treatment efficacy in bacterial keratitis. Expert Opin Drug Deliv. 2015;12(11):1791-807.

Wang L, He H, Zhang C, Sun L, Liu S, Yue R. Excellent antimicrobial properties of silver-loaded mesoporous silica SBA-15. J Appl Microbiol. 2014;116(5):1106-1118.

Received for publication on $17^{\text {th }}$ April 2018 Accepted for publication on $16^{\text {th }}$ May 2018 